\title{
Fricción del espacio y externalidades territoriales de la movilidad turística terrestre en América Central
}

\author{
Gino Jafet Quintero Venegas* Álvaro Sánchez Crispín** \\ Universidad Nacional Autónoma de México (México)
}

\begin{abstract}
Resumen: El turismo se ha convertido en una de las fuerzas motrices del proceso de integración centroamericana contemporánea y, como resultado, se ha consolidado la ruta de transporte terrestre Ticabus (RTT). La RTT es un recorrido terrestre que vincula a Tapachula, México, con la ciudad de Panamá a través de la Carretera Panamericana. Con $2526 \mathrm{~km}$ de longitud, cruza por seis de los siete países del istmo centroamericano y con excepción de dos localidades (Tapachula, México y San Pedro Sula, Honduras), los puntos de origen-destino son las capitales nacionales de América Central. Sin embargo, a pesar del tamaño de los países, de la presencia de infraestructura carretera y del vínculo directo entre capitales nacionales, hay una serie de externalidades territoriales, dadas por condiciones políticas, económicas y sociales que potencian la fricción del espacio y evidencian la dificultad del dinamismo territorial en América Central.
\end{abstract}

Palabras Clave: Fricción del espacio; Integración turística; Transporte turístico; América Central.

\section{Space friction and territorial externalities in overland tourism mobility in Central America}

Abstract: Tourism has become one of the driving forces of the contemporary integration process in Central America and, as a result, the land transport route Ticabus (TRT) was developed. This route links Tapachula, Mexico to Panama City through the Pan American Highway and and crosses six of the seven Central American countries in $2526 \mathrm{~km}$. With the exception of two locations (Tapachula, Mexico and San Pedro Sula, Honduras), the points of origin and destination are national capitals of Central America. Despite the small size of the countries, the presence of the highway infrastructure and the direct links between national capitals, there are a number of territorial externalities, as a result of political, economic and social conditions, which not only maximize the friction of space but also demonstrate the difficulty of territorial dynamics within the isthmus.

Keywords: Space friction; Tourist integration; Tourism transport; Central America.

\section{Introducción}

El presente trabajo reflexiona sobre el papel que ha jugado el territorio como modelador de la integración turística centroamericana. Su objetivo es explorar la situación actual del turismo centroamericano y cómo las externalidades generadas son un desafío para que América Central se pueda posicionar como una región unificada en el contexto internacional. Para ello, toma como base la premisa de que el turismo ha sido una estrategia significativa de crecimiento para la mayoría de los países de Centroamérica, los cuales han cambiado su estructura económica, del sector primario al sector terciario, en los últimos veinte años (Pigliaru y Lanza, 1999).

El modelo de turismo de masas, implementado en los países ístmicos, ha sido ampliamente criticado por sus escasos e incipientes resultados en el desarrollo económico: no ha contribuido a mejorar la calidad de vida de la población local y ha favorecido la acumulación de capital de los grandes corporativos a costa de los recursos locales (Liu y Jenkins, 1996). Además, este modelo es rechazado por los visitantes actualmente

\footnotetext{
* Candidato a doctor en Geografía por parte de la Universidad Nacional Autonoma de Mexico; E-mail: england_km@hotmail.com * Departamento de Geografía Económica del Instituto de Geografía, Universidad Nacional Autonoma de Mexico; E-mail: ascrispin@yahoo.com
} 
porque se buscan actividades más auténticas y enmarcadas dentro del turismo alternativo (Wang, 1999). Así, las instituciones turísticas centroamericanas, como el Sistema de Integración Centroamericana (SICA), el Consejo Centroamericano de Turismo (CCT) o los ministerios nacionales de turismo, han creado un producto turístico centroamericano basado en la arqueología maya, la biodiversidad y las playas, orientado hacia viajeros jóvenes y alocéntricos ${ }^{1}$ quienes no temen desplazarse a través del territorio (Lew y McKercher, 2006).

El turismo centroamericano es visto como una alternativa doble para el crecimiento económico regional: es una actividad formal generadora de empleos y captadora de divisas y se ve como una opción viable para estabilizar la balanza de pagos y abatir la dependencia de las exportaciones, generalmente de materias primas (Brohman, 1996; Liu y Jenkins, op. cit.). Además, se considera una actividad que puede reducir la pobreza y crear trabajos que financien a las clases marginales. No obstante, al igual que cualquier actividad económica, es altamente sensible al ambiente político y cultural en que se desenvuelve (Buckley, 2003).

La actividad se ha promovido y financiado tanto por sectores públicos y privados, como por nacionales e internacionales (Ferguson, 2010). Sus fondos sectoriales de inversión tienen como base la reducción de la pobreza (Cañada, 2010; Ferguson, op. cit.). Además, es compatible con los Objetivos de Desarrollo del Milenio de la ONU, porque busca integrar a las poblaciones marginadas y promover la igualdad de género y autonomía de las mujeres (Escaith, 2001; ONU, 2005).

Desde que el turismo se ha visto como un motor de crecimiento económico para los países subdesarrollados, los trabajos que se han realizado sobre la conformación de rutas turísticas en América Latina han tenido una gran profusión y alientan la creación de rutas soportadas en elementos naturales o culturales (López y Sánchez, 2008); sin embargo, la mayoría de estos trabajos refieren a rutas infalibles y sin obstáculos y a estrategias para poder consolidar la integración sin tomar en cuenta fallas y debilidades presentes en el territorio. López y Sánchez (op. cit.), por ejemplo, hablan de la importancia de crear rutas en función de componentes que resalten las características locales y regionales; Fernández y Ramos (2005), a través de sus estudios sobre patrimonio turístico industrial aseveran que la creación de rutas turísticas permite la reactivación de las economías locales; mientras que Briedenhann y Wickens (2003) sostienen que tanto el agrupamiento (clustering) de las actividades y atracciones como el desarrollo de rutas de turismo estimulan la cooperación y las alianzas entre las áreas locales.

A pesar de que la ruta turística Ticabus de América Central no ha figurado particularmente como objeto de estudio académico, su pertinencia se soporta no sólo porque es la médula espinal de la movilidad terrestre regional sino porque refuerza la importancia del vínculo entre el transporte terrestre con la actividad turística. Por ejemplo, Lew, Hall y Williams (2008) hablan del papel que juegan el auto y los autobuses como parte fundamental del viaje recreativo; Lumsdon (2000) asevera que el transporte no es un componente de la oferta turística sino un medio, mientras que López y Sánchez (2008), mencionan que la actividad turística no podría ni materializarse ni generar los flujos de personas actuales sin la presencia de transporte e infraestructura.

Las estrategias metodológicas empleadas en este artículo para cubrir el objetivo central de develar las externalidades territoriales fueron de carácter cualitativo. Durante las cuatro vistas a campo hechas entre 2013 y 2015 se realizó observación in situ participativa, observación no participativa y entrevistas polilógicas ${ }^{2}$ (Anderson, Adey, Bevan, 2010) al interior de los autobuses que conforman la ruta del Ticabus. Además, se trabajó con micro-etnografías particularistas para focalizar el trabajo de campo a través de la observación e interpretación del fenómeno de la movilidad turística en un contexto cotidiano de movilidad.

Tedlock (2010) argumenta que un investigador puede ser parte del universo a estudiar como parte de las narrativas etnográficas; así, se fungió como turistas usuarios del Ticabus para vivir y percibir el espacio de movilidad centroamericano y valorar el papel la integración a partir de experiencias personales y colectivas (Murillo y Martínez, 2010). Para complementar la información recabada de forma directa en campo, se usó el método del análisis multimodal del discurso virtual (Levine y Scollon, 2004), una técnica de investigación emergente que consiste en clasificar los datos obtenidos de las redes sociales y de los foros de discusión virtuales utilizados por los turistas en América Central utilizan para emitir opiniones respecto a los medios del transporte en la región; se escogió este método porque, como mencionan Belz y Baumbach (2010), su uso se basa en el hecho de que, en la actualidad, la comunicación virtual es una de las manifestaciones más influyentes cuando los viajeros toman decisiones. El texto se organizó en tres partes para cubrir los objetivos de la investigación: en primer lugar, se hace un recuento de las políticas de integración centroamericana, desde finales del siglo XX y su asociación con el transporte terrestre; la segunda parte se centra en clasificar los diferentes obstáculos territoriales que potencian la fricción del espacio, concepto que se explicará posteriormente, e imposibilitan la integración regional centroamericana; y en la tercera parte se discute sobre algunas consideraciones espaciales que deberían tomar las instituciones para que se logre la integración. 


\section{El desarrollo del turismo y la integración regional centroamericana}

La implementación del turismo centroamericano se sustenta en dos instrumentos políticos: la Declaración de Montelimar, firmada en 1996, que reconoce la fuerza que tiene la actividad para mejorar la competitividad global del istmo y aumentar la diversificación de las economías nacionales; y las creación de las diez Secretarías Técnicas Especializadas del Sistema de Integración Centroamericana, encargadas de la integración política y económica en América Central (Solís, 2014; Ullate, 2014).

La consolidación de una región turística ha sido el intento de integración más exitoso en América Central. Esta entidad territorial ha favorecido que se formulen políticas y se instauren instituciones para alcanzar el desarrollo económico (Romanelli y Khessina, 2005). Por ejemplo, la Secretaría de Integración Turística Centroamericana (SITCA), el órgano responsable de elaborar y efectuar las políticas turísticas de la región, trabaja estrechamente con el Cuerpo de Integración de América Central y con el Cuerpo de Competitividad y Desarrollo Regional. Esta relación político-laboral ha facilitado la promoción turística del istmo -como la Ruta Colonial y de los Volcanes-, y desarrollar el vínculo con el transporte terrestre (SICA, 2013). También, el Consejo Centroamericano de Turismo (CCT), órgano compuesto por los siete ministros de turismo nacionales, aborda el desarrollo del turismo:

"[El objetivo es] promover el desarrollo del turismo en toda la región de América Central con el fin de integrar a la industria (sic.) como un sector estratégico en cada país, con el objetivo de contribuir al desarrollo del turismo sostenible en la región. Además, [...] facilitar y estimular el desarrollo del turismo en toda América Central, a partir de la eliminación de cualquier obstáculo e impedimentos a la libre circulación de personas en la región y la integración de la promoción del turismo, como un papel del Estado, en todas las actividades de las diferentes ramas de gobierno" (SICA, 2013).

Los intereses del sector privado han estado cubiertos en el plan de integración turística centroamericana desde el principio. El proyecto se originó como respuesta a la presión de los actores turísticos que deseaban invertir o expandirse en la zona. SITCA ideó como estrategia política de integración la incorporación del sector privado dentro del sector público al momento de la toma de decisiones en la Declaración de San José de 2002. Además, la política de desarrollo turístico está influenciada por los objetivos tanto de los capitales turísticos regionales e internacionales como de los intereses de dichos actores, han quedado representados en los procesos políticos de integración (Ferguson, 2010; Hale, 2011).

América Central ha recibido apoyo del exterior para concretar la integración a partir del turismo. La Agencia Suiza para la Financiación del Desarrollo y de la Cooperación vincula al istmo con el Centro de Información Global sobre la Financiación para el Desarrollo. Ambas organizaciones han facilitado la inversión del sector turístico privado y de transporte en los países del istmo (Ferguson, 2010; Jiguan, 2015).

A pesar de todos los mecanismos internacionales de inversión y apoyo por parte de instituciones privadas y gubernamentales, América Central se enfrenta a ciertos desafíos para consolidar su integración regional. El primero de ellos es la acción colectiva y multinacional para construir la integración en forma simultánea con miras a la inserción en una economía global. Por ejemplo, hay una contradicción económica y espacial que, aunque existe la marca "Centroamérica, tan pequeña, tan grande", los países compiten entre ellos por la mayor captación de turistas en un contexto de oferta de recursos similares (SICA, 2013).

Tras la inestabilidad política y social de fines del siglo pasado, los países centroamericanos enfrentan desafíos en la construcción de la democracia, la participación, la modernización de la economía y la inversión en infraestructura carretera -elemento fundamental para el desplazamiento turístico-. Así, cuando las vías de comunicación están en buen estado, el tránsito vial refleja unidad regional (Reid, 2007) y cuando están deterioradas, se dificulta la movilidad de capitales, personas y mercancías. Con base en esto, la toma de decisiones colectivas en torno a la movilidad y al estado de las carreteras es una pieza central de la integración que debe fortalecer los objetivos estratégicos como la decisión de seguimiento y coordinación, la aplicación de las decisiones subregionales a nivel nacional, la comunicación y la participación de la sociedad civil en el proceso de integración, y las relaciones exteriores y cooperación.

Actualmente, la movilidad territorial es un nicho de oportunidad política y económica para lograr la integración. El Acuerdo de Control Fronterizo Centroamericano (CA-4), firmado en junio de 2006 entre El Salvador, Guatemala, Honduras y Nicaragua, establece la libre circulación de personas sin ningún tipo de restricciones o controles, a través de las fronteras de los cuatro estados miembros. Los extranjeros que entran en los países del CA-4 pueden desplazarse por tierra entre ellos sin la necesidad de obtener permisos adicionales o de someterse a controles en los puestos fronterizos. Al igual que en el Acuerdo de Schengen en Europa, el Acuerdo CA-4 establece un régimen de visados armonizados para 
los extranjeros que se movilizan por la zona (Segovia, 2005). Sin embargo, esto es más un discurso que una realidad debido a las externalidades territoriales presentes a lo largo del istmo.

\section{Las externalidades territoriales y la fricción del espacio como obstáculos de la integración regional}

Las externalidades son decisiones de consumo, producción e inversión que toman los individuos, los hogares y las empresas y que afectan a terceros que no participan directamente en esas actividades comerciales (Berliant, Peng y Wang, 2002). Laffont (2011) las define como aquellas situaciones en que los costos o beneficios de producción y/o consumo de algún bien o servicio no son reflejados en el precio de mercado de los mismos. Mion (2004) y Fujita y Krugman (2004), sostienen que son fallas de mercado y efectos externos que producen ineficiencia, obstaculizan y atenúan el desarrollo de alguna actividad económica. En función del grado de afectación hacia la dinámica económica, éstas son negativas o positivas (Mion, op cit.; Hagoort, Geertman, y Ottens, 2008).

Cuando la lógica económica del mercado y de las actividades económicas no se extiende a todos los recursos y servicios, surgen externalidades negativas. Éstas no son culpa del mercado, sino de la intervención del Estado o de su imposibilidad de sortear con elementos naturales, como la orografía del sitio la presencia de fenómenos naturales. Las externalidades negativas impiden el desarrollo adecuado de cualquier actividad económica y repercuten tanto en la falta de planificación territorial como en la firma de acuerdos que conlleven a una verdadera integración regional (Navalpotro, 2001). Por otro lado, las externalidades positivas emanan cuando acciones empresariales públicas o privadas benefician tanto a los agentes directamente implicados en ellas como a los free-riders ${ }^{3}$ (Anselin, Vargas y Acs, 2000). Para el caso concreto del turismo centroamericano, la mejora de las carreteras y de los servicios de transporte turísticos ocasiona que la población local vea un aumento en la eficiencia de la movilidad y se facilite el comercio y el tránsito regional a través del istmo. Este tipo de externalidades se respaldan en la teoría de la no exclusión de los bienes públicos -se produce para todo el mundo o para nadie- y no hay rivalidad en el consumo -el que una persona consuma de ese bien no hace que haya menos para los demás-(Anselin, 2003).

La pauta en que se distribuyen las actividades en los territorios es un tema recurrente en geografía y en economía. Sin embargo, no hay un consenso general de cómo el territorio repercute en el desarrollo de una actividad; para Krugman (2011) y Glaeser y Scheinkman (2000), el territorio funciona como soporte y como proveedor de los recursos; mientras que para Becattini (2002) es sólo una relación que se establece entre poder y espacio. En este artículo, el territorio se entiende a partir de la concepción de Krugman (op. cit.) y Glaeser y Scheinkman (op. cit.) y, por lo tanto, posee características intrínsecas como la dotación de recursos naturales, la accesibilidad y los factores institucionales. El grado de inmovilidad del capital físico, las diferencias socioculturales a diferentes escalas y los agentes antrópicos y naturales que imposibilitan el desarrollo pleno de la actividad turística se conciben como las externalidades territoriales.

Las externalidades territoriales son tema en curso en el análisis económico porque repercuten en el crecimiento y en el desarrollo regional y deben considerarse cuando se proponen proyectos de integración (Glaeser y Scheinkman, 2000). En América Central, la relación turismo-transporte se ha visto mermada por externalidades territoriales que conllevan a que los costos y los obstáculos presentes en la actividad no igualen los beneficios del desarrollo de la infraestructura carretera y de los planes y acuerdos de integración regional. Debido a la presencia de estas externalidades a lo largo de la RTT, se asevera que el espacio centroamericano no está acondicionado para brindar movilidad terrestre intrarregional eficiente por la presencia de elementos naturales, sociales, políticos y económicos como las condiciones físicas de las carreteras, el relieve accidentado del istmo, la carencia de rutas alternativas, la presencia de fronteras poco eficaces, la calidad del transporte y los manejos administrativos. Todas, en su conjunto, aumentan la fricción del espacio, concepto utilizado cuando se prolongan los tiempos de viaje aunque las distancias recorridas sean cortas (Puebla, 1998).

\section{La fricción del espacio en América Central}

Un requisito básico para la evolución del comercio internacional y del turismo inter y transnacional es el desarrollo de tecnologías -transporte y comunicaciones- que superen el efecto de la fricción del espacio. En la era global, los lugares se relacionan e interconectan de manera instantánea como resultado en las mejoras en las tecnologías de la información y la comunicación (Fujita, Krugman y Venables, 2001). Cuando se logra la interconexión, los países que le han apostado al turismo como el motor de crecimiento económico, se pueden insertar en el complejo sistema mundial. 
Como estrategia de inserción en el sistema mundial, se implementó en Centroamerica la ruta terrestre del Ticabus, de más de $2500 \mathrm{~km}$ y que se recorre a una velocidad promedio de $17 \mathrm{~km} / \mathrm{h}$. A pesar de que esta velocidad tan baja significa que existe una fricción del espacio muy alta porque el tiempo para desplazarse por toda la ruta es de 147 horas, los viajeros que visitan América Central deciden utilizar el transporte terrestre porque es una alternativa económica viable al no existir aerolíneas de bajo costo que vinculen las distintas capitales de la región (Trip Advisor, 2016). No obstante los tiempos de desplazamiento, el trabajo de campo reveló que la ruta es exitosa porque es utilizada por personas jóvenes, latinoamericanos y con bajos ingresos, cuyos viajes son del tipo de turismo mochilero y quienes son, además, el principal target mercadológico de la industria turística centroamericana.

Sin embargo, la fricción del espacio repercute en la integración regional porque funciona como filtro de la movilidad terrestre. Los patrones de consumo y comportamiento de los turistas se merman porque no todos los visitantes están dispuestos a viajar distancias cortas en tiempos prolongados y a sortear la serie de vicisitudes territoriales que transforman al viaje en un periplo (Suzuki, 2010). De acuerdo con lo obtenido en campo, aquéllos que están dispuestos a realizar los largos recorridos de la ruta son personas jóvenes que ven al desplazamiento como parte de la aventura del viaje; tienen ingresos más bajos y menos experiencias de viaje. Por otro lado, los turistas de mayor edad prefieren recorrer distancias más cortas y en menos tiempo, tienen mayores ingresos económicos, son más experimentados y optan por actividades en donde haya confort y que se vincule con sitios que están clasificados como Áreas Naturales Protegidas. Este descubrimiento en campo se complementa con la idea de McKercher y Lau (2008) acerca de que cualquiera viaja distancias largas, aunque hay tamices de exclusión como el exceso en los tiempos de deslazamiento, los costos del viaje, el esfuerzo físico y la voluntad para entrar en ambientes culturalmente distintos.

A pesar de que ha habido esfuerzos en conjunto de los países centroamericanos por mejorar el transporte terrestre regional y han invertido en carreteras de forma colectiva, aún hacen falta acciones que reduzcan los tiempos de desplazamiento. La ineficacia de la movilidad en los cruces fronterizos, la corrupción y el racismo son algunos de los obstáculos a sortear, y la mayoría de los usuarios del Ticabus les asocia con un alto costo a pagar por disfrutar del viaje a través de la región. Aunque el Ticabus ha mejorado la movilidad regional desde los años setenta, la fricción del espacio no ha disminuido porque la ruta no es directa desde Tapachula hasta Panamá; los viajeros deben pernoctar en cada una de las capitales centroamericanas y después, continuar el recorrido. Debido a esta situación, el $40 \%$ de los usuarios del transporte deciden no realizar la totalidad del recorrido porque gastan parte importante de su presupuesto temporal en las escalas y del económico en hospedaje. Esto puede generar frustración en el viaje porque no se logra recorrer todo el istmo ni se visitan todos los sitios emblemáticos.

\section{Externalidades políticas}

Este tipo de externalidades son el resultado de procesos históricos y de elementos sociales y culturales propios de la región. Las primeras de ellas son las fronteras internacionales, barreras físicas que limitan el contacto entre personas y funcionan como líneas de contención y de defensa militar. También son planos verticales invisibles que seccionan el espacio aéreo, el suelo y el subsuelo entre países colindantes y marcan el límite del territorio en el que un Estado puede ejercer su autoridad soberana (Rudolph, 2005). Timothy (1995) sugiere, por ejemplo, que los pasos fronterizos funcionan como embudos y concentran el tráfico de áreas amplias en ambos lados.

La separación generada por los cruces fronterizos repercute en las percepciones de los turistas y de la población local sobre el espacio centroamericano y su grado de integración (Rudolph, op. cit.). Además, juegan un papel dual en la movilidad porque están hechos para separar y para unir al mismo tiempo: delimitan y son traspasados y diferencian y diluyen diferencias sociales y económicas (Timothy, op. cit., Paasi, 2009). En el turismo contemporáneo, cruzar una frontera es sinónimo de acumular una experiencia, aunque también de incertidumbre y de nerviosismo:

"Cada cruce de fronteras es lo mismo; esa sensación de orfandad, los nervios, el mismo espacio como de vacío, que parece no existir, de gente en tránsito, de gente buscándose la vida, turistas, viajeros, mochilas, pasaportes, visados..." (Entrevistado durante el tercer recorrido de campo, 2014).

Otra valoración de las fronteras radica en su noción de líneas de contacto: son lugares donde convergen culturas similares y diferentes (Timothy, op. cit.; Paasi, op. cit.) y pueden generar a problemas de territorialidad y movilidad. Por sí mismas, las fronteras son un recurso turístico aunque los trámites asociados con los cruces internacionales son molestos y aumentan la distancia percibida en ciertos destinos (Timothy, op. cit.). 
El control de ingreso y salida de personas en un país, la revisión de pasaportes y las inspecciones aduaneras son también externalidades políticas. De acuerdo con el acuerdo CA-4, hay libre tránsito por el istmo centroamericano y facilidad para realizar los trámites en las fronteras (Segovia, op. cit.). No obstante, la falta de infraestructura aduanera, los conflictos fronterizos la región y la presencia del narcotráfico, han hecho que tanto en la frontera entre Nicaragua y Costa Rica como en la de Honduras y El Salvador, los turistas permanezcan en los autobuses al momento de hacer los trámites fronterizos. En todas las fronteras terrestres se realiza un estricto control de revisión de equipaje, de forma manual, que obstaculiza la movilidad regional. Allí se exige a los usuarios del autobús a descender de la unidad y surgen relaciones de poder entre locales y extranjeros porque los últimos son interrogados (Figura 1).
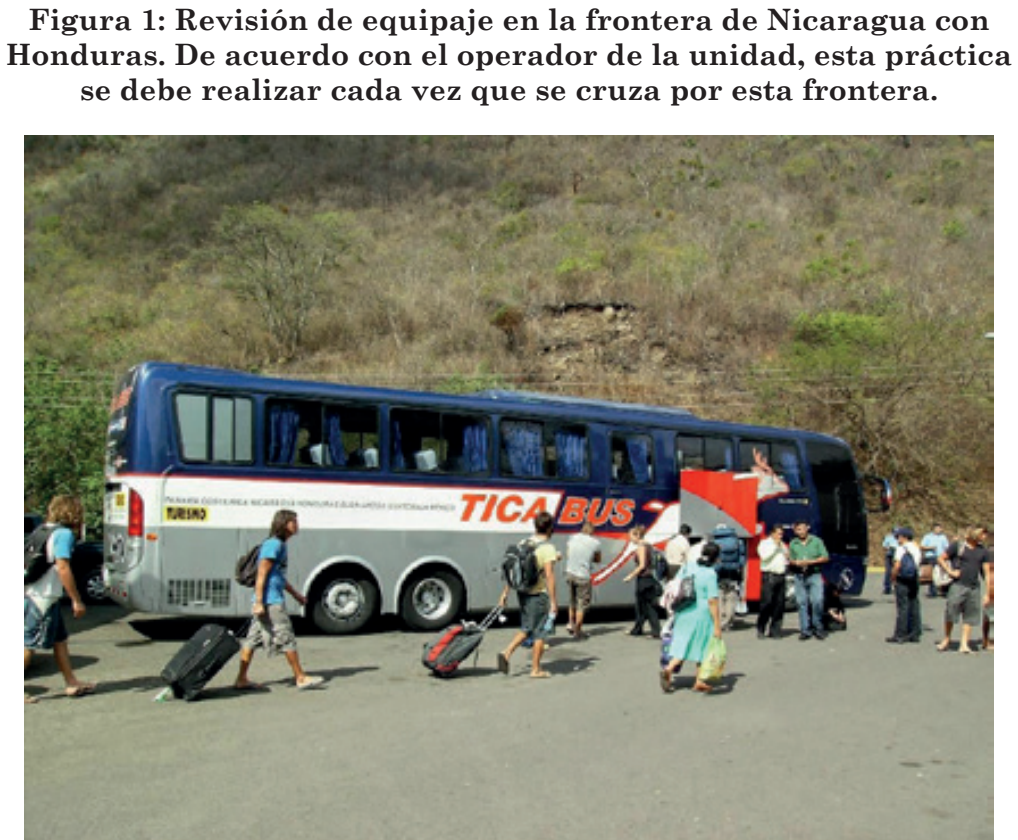

Fotografía: Quintero, trabajo de campo 2015.

El racismo y los prejuicios étnicos interfieren tanto en la movilidad territorial como en el desarrollo de la actividad turística centroamericana. Por ejemplo, los agentes aduanales valoran arbitrariamente a las personas en función del fenotipo y del color de piel; para aquellos visitantes con apariencia caucásica y con pasaportes sellados por otros países el trámite es muy sencillo y eficaz y se les permite el acceso por el istmo sin algún impedimento, y a quienes tienen aspecto mestizo o indígena se les cuestiona exhaustivamente sobre los motivos del viaje. Este tipo de discriminación turística en las fronteras de istmo hace que el tiempo estimado para realizar trámites migratorios sea de una hora.

Hay fuertes problemas de movilidad a través de América Central por lo ambiguo de los visados. La permanencia a ser otorgada por el segundo país o posterior en que se lleva a cabo el desplazamiento, no debe ser mayor a la duración del visado otorgado por el país inicial de ingreso a la región (Prideaux, 2005; Go Central America, 2016). Sin embargo, cada país suele poner un sello en el pasaporte sin realmente contar con los días de estancia en algún país vecino o, como en el caso de El Salvador, las restricciones de entrada son muy fuertes por ineficiencias del control migratorio y de la comunicación con otras fronteras. Ante esto, los turistas se sienten confundidos y su percepción del istmo radica, principalmente, en la falta de integración regional y en los fuertes revanchismos nacionales (Robinson, 2003).

\section{Externalidades económicas}

La calidad de las carreteras es un sello de garantía para el crecimiento y la integración regional porque son el soporte de la movilidad terrestre. No obstante, a pesar de buscar insertarse en el mercado 
turístico internacional, algunos países centroamericanos no han invertido ni en la ampliación ni en el mantenimiento de sus carreteras, situación que dificultan la movilidad y la integración regional. Además, se genera una contradicción in situ del crecimiento económico porque no hay relación alguna entre el estado y la calidad de la infraestructura vial y el grado de desarrollo. Los países con las economías turísticas más competitivas en el istmo, como Costa Rica y Guatemala, por ejemplo, presentan serios problemas en sus carreteras; mientras que aquéllos donde hay menor calidad de vida, como Honduras y Nicaragua, han construido y mantenido en buen estado sus vialidades como resultado de tratados internacionales como el CA-4.

El mal estado de las carreteras es una constante en casi toda la región (Figura 2). Aún falta mucha inversión en para darles mantenimiento y mejorarlas y que realmente sean funcionales y respondan a la creciente demanda de transporte local e internacional. Así, como sostiene Mora (2013), la mejora de la infraestructura vial y la eficiencia y transparencia de las fronteras son los principales retos de la integración centroamericana:

"Si pretendemos una regionalización, éstos son los principales cuellos de botella que tenemos que resolver: los pasos fronterizos y las condiciones de las vías para que puedan transitar de manera segura los usuarios y las mercancías [...] En muchos casos no son vías seguras o en buenas condiciones" y agrega que en los pasos fronterizos "muchas veces la mercancía pasa mucho tiempo en procesos demasiado largos para poder pasar de país a país, lo que aumenta costos y hace menos eficiente la actividad económica".

Figura 2: América Central: externalidades territoriales y fricción del espacio

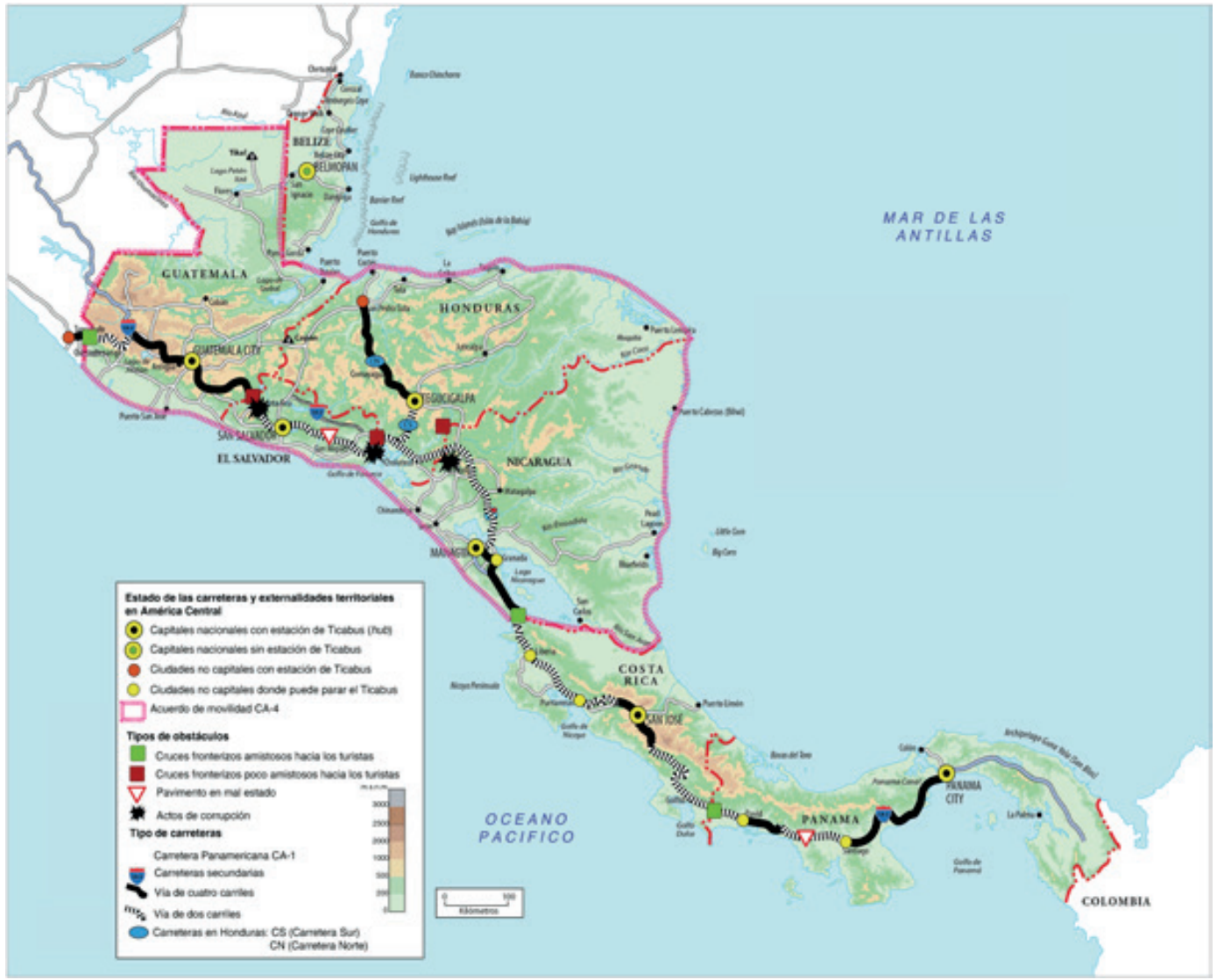

Fuente: elaboración propia con base en trabajo de campo 2013-2016. 
Otra externalidad económica a la que se enfrentan los turistas en la región es la dificultad para cambiar divisas y monedas nacionales. Belize, Guatemala, Honduras, Nicaragua y Costa Rica tienen su propia moneda nacional, mientras que El Salvador y Panamá utilizan el dólar estadounidense. Los costos de transacción que resultan de cambiar monedas cada vez que se cruzan las fronteras, gracias al valor de compra y de venta, han hecho que la divisa norteamericana se acepte en casi todos los sitios turísticos de América Central. Incluso, los cajeros automáticos de Nicaragua y Belize, dispendian dólares bajo petición. La excepción a este fenómeno de semidolarización es Guatemala por tener una política económica proteccionista hacia el quetzal (Bahl, Martínez y Wallace, 1997).

No obstante, pese a la amplia aceptación del dólar, las monedas locales se prefieren para pagos de bienes y servicios menores a veinte dólares, como alimentación, pago del transporte local o entradas a museos. La aceptación del dólar es una externalidad negativa de la integración porque se prefiere ante las monedas locales y eso repercute en la inflación (Rodríguez y Dombrow, 2016). Cuando se transita entre países, los viajeros deben deshacerse del excedente de moneda local en los pasos fronterizos porque en las ciudades de llegada del autobús es poco probable encontrar casas de cambio que acepten monedas centroamericanas. Si el Banco Centroamericano de Integración Económica introdujera oficialmente una moneda común para el istmo, se favorecería la movilidad y la integración; sin embargo, la tendencia hacia la dolarización ha evitado crisis cambiarias y de las balanzas de pagos a lo largo del istmo (Agencia Española de Cooperación Internacional, 2002).

\section{Externalidades socioculturales}

Las externalidades socioculturales repercuten en la movilidad de forma individual en cada viajero. El tiempo es uno de los pocos factores inamovibles a los que se enfrentan los turistas cuando viajan. Los tiempos de vacaciones son usualmente fijos y con pocas posibilidades de extensión. Sin embargo, los viajeros controlan sus presupuestos de tiempo, ponderan el recurso y valoran el costo del viaje: o se percibe como escaso y debe racionalizarse o se puede gastar en diversos lugares (McKean, Johnson y Walsh, 1995).

Cuando el tiempo de viaje es inamovible, aquél empleado para el tránsito representa un costo porque el gastado en la movilidad debe cambiarse por una estancia más corta en el lugar de destino (Truong y Henscher, 1985). Si hay poca movilidad intradestino, los turistas maximizan el tiempo en los destinos finales y minimizan los tiempos de tránsito (Lew y McKercher, 2006). Las decisiones personales de movilidad generan una disyuntiva en donde, tanto los tiempos de desplazamiento desde el sitio de origen al lugar de destino como los desplazamientos intradestino, son tan significativos como el sitio de destino y la movilidad, y la observación de los paisajes durante los recorridos [sightseeing] y las diferentes escalas se vuelven igual de importantes.

Fakeye y Crompton (1991) aseveran que hay diferencias sustanciales en la movilidad, el número de actividades a realizar y la percepción de la integración entre viajeros primerizos y aquellos que repiten estancia a partir de sus características culturales personales. Los primeros tienen más probabilidades de hacer viajes prolongados y cruzar fronteras nacionales para incrementar su capital espacial. Los repetidores suelen hacer incursiones cortas e intermitentes y regresan al lugar de alojamiento durante el día (McKercher et al., 2003). La duración de una escala y la inversión psicológica de la movilidad influyen también en el patrón de movilidad dentro del lugar visitado (Li y McKercher, 2016).

El motivo del viaje afecta la distribución espacial de los turistas y repercute en la integración regional cuando existen polos de desarrollo (Sunkel, 1998). Los paseantes hedonistas suelen explorar más que aquéllos que viajan por negocios. Aquéllos que transitan por motivos familiares se movilizan menos pues prefieren pasar tiempo con sus conocidos (Pearce y Moscardo, 2006; Griffin, 2013) y suelen desplazarse sólo hacia los nodos turísticos y los destinos preferenciales. Quienes tienen intereses espaciales particulares acotan sus desplazamientos a actividades relacionadas con sus propios motivos del viaje (como los turistas de buceo en América Central), y quienes tienen interés mixtos se desplazan más ampliamente sin patrones evidentes de movilidad y disfrutan de observar los paisajes urbanos, rurales y naturales. Estos últimos son quienes viven visual y sensorialmente el espacio y perciben más objetivamente la integración centroamericana (Zakrisson y Zillinger, 2012).

Los operadores del Ticabus también son agentes que ejercen poder hacia las decisiones de movilidad de los turistas y son los responsables de una externalidad sociocultural: las escalas. Ellos deciden arbitrariamente los tiempos para bajar a comer, "estirar las piernas" y salir a respirar aire fresco. Hacer una escala se decide por el tiempo de desplazamiento y no por la distancia recorrida y significan retrasos en el viaje, tiempos perdidos y momentos de traslado anodinos que pueden generar frustración en los viajeros. 
Las externalidades socioculturales también dependen de la estabilidad de los sistemas políticos. Cuando hay estabilidad política se invierte más en infraestructura (hoteles, carreteras, estaciones de transporte) y se amplían los servicios turísticos (campañas de mercadotecnia y seguridad) (Khadaroo y Seetanah, 2008). La inversión turística no sólo promueve el crecimiento económico sino que crea la imagen de que el destino es un sitio cómodo y seguro para viajar y movilizarse (Hall y O'Sullivan, 1996). Cuando un país experimenta convulsiones políticas o incidentes relacionados con terrorismo o corrupción, generalmente se les advierte a los turistas potenciales que no lo visiten y se merma la llegada de visitantes y la captación de divisas.

Si en un país hay prácticas de soborno o negocios fraudulentos, se afecta negativamente la actividad turística porque se deteriora la imagen cultural y social del destino y porque se impide su competitividad en el sistema mundial (Das y DiRienzo, 2010; Lau y Hazari, 2011). La corrupción es una externalidad sociocultural ya que genera barreras para las inversiones en el turismo (Tosun y Timothy, 2001), disminuye los flujos turísticos hacia los espacios donde se generan y exacerba los conflictos sociales porque hay choques de intereses entre los agentes políticos y la población local (Nieto, 1996). Sin embargo, la corrupción representa una dualidad en la movilidad turística centroamericana: hay visitantes motivados a desplazarse hacia el istmo porque perciben que los altos índices de corrupción son parte de la experiencia del viaje y de la vivencia del lugar (Mires, 2006) y causa efectos adversos al modificar las decisiones de los turistas para desplazarse a través de la región (Issa y Altinay, 2006).

Una recomendación que hacen los gobiernos de ciertos países europeos a sus conciudadanos para viajar a América Central es "rehuir de cualquier problema oficial o policial innecesario" ya que la situación política en la región, principalmente con referencia en la presencia de los Maras Salvatrucha y el narcotráfico, es "compleja desde hace décadas y la corrupción se asienta en algunas vertientes de la administración pública y de los servicios oficiales". Wikitravel y Trip Advisor advierten, también que "el sistema legal [centroamericano] no está bajo ningún elemento del código Napoleónico". Además, con respecto a la corrupción y la seguridad en el istmo, agregan, que:

"los oficiales de tránsito pueden usar un pequeño pretexto para detener a los turistas y multarlos. Si es detenido por un oficial que exige un soborno, no le pague la cantidad exigida, dígale que no tiene dinero en efectivo porque si usted saca dinero y lo da, es corrupción y como tal es considerado un crimen en el país, por lo que es una decisión personal si lo hace o no. La multa por ir a exceso de velocidad puede ser de aproximadamente 100 dólares, dependiendo la ciudad. Los criminales pueden posar como policías, así que manténgase siempre en un lugar visible, en público, y en su coche”. (NVI Noticias, 2011)

"respete las leyes regionales. Muchos extranjeros piensan que América Central es un lugar donde las leyes pueden romperse y la policía sobornada en todo momento. La corrupción puede ser común en la policía o figuras públicas. Cuando los foráneos actúan de manera que esperan un soborno fácil, es considerado irrespetuoso y puede ser usado con pretexto por la policía para darte una lección de respeto. Recuerda que ofrecer un soborno a un oficial puede meterte en más problemas en lugar de evitarlos" (Wikitravel, 2016).

"si viaja en autobús, no ponga objetos valiosos en su equipaje grande. En las revisiones de la policía o militares pueden tomar lo que necesiten, sobre todo en los viajes nocturnos cuando los pasajeros vienen dormidos (Trip Advisor, 2016)”.

Para que la integración y la movilidad no se vean afectadas a partir de la externalidad tan negativa de la corrupción, los gobiernos centroamericanos deben resolver los problemas en torno a ella.

La inseguridad y la violencia también son externalidades que afectan la movilidad y que se relacionan directamente con la corrupción. A pesar de que la inseguridad en las carreteras ha disminuido en los últimos años, el tema se mantiene como un problema de facto y perturba la decisión de desplazamiento de los visitantes. Los servicios de transporte Premium -como el Ticabus-, no son necesariamente la opción más segura como lo atestigua una de las usuarias:

"A mí me asaltaron en agosto 2011 regresando de Guatemala en el bus [...]. Estábamos de vacaciones con mis hijos en El Salvador y habíamos ido a que conocieran Guatemala junto con sus novias (italianas). Nos asaltaron cuando el bus estaba por llegar a la frontera de El Salvador. Se atravesaron dos jeeps cortándole el camino al bus y como ocho hombres enmascarados y armados de pistola salieron de los matorrales y se subieron al bus gritando y amenazando. Nos robaron todo pero gracias a Dios no hubieron heridos (a parte un golpe con la pistola en la cabeza al señor que estaba en frente de mí y una jalada de pelo a una señora que estaba escondiendo su cartera). (Testimonio en campo)" 
Estados Unidos ha intervenido económicamente en la región para erradicar la corrupción a partir de la Iniciativa Regional de Seguridad para América Central (CARSI, por sus siglas en inglés). Éste es un programa de colaboración birregional para mejorar la seguridad ciudadana (CARSI, 2012) y ha propuesto cinco objetivos que mejoren la integridad tanto de los habitantes locales como de los turistas:

1) Crear calles seguras para los ciudadanos de la región;

2) Desbaratar el movimiento de los criminales y el contrabando en y entre los países centroamericanos;

3) Apoyar el desarrollo de gobiernos fuertes, capaces y responsables en América Central;

4) Restablecer la presencia efectiva del Estado, los servicios y la seguridad en las comunidades en peligro; y

5) Fomentar mayores niveles de coordinación y cooperación entre los países de la región, otros asociados internacionales y donantes para combatir las amenazas a la seguridad regional.

Los objetivos tienen una finalidad doble: que los países centroamericanos aumenten su seguridad y la prosperidad de sus ciudadanos, y se establezcan instituciones responsables que promuevan los derechos humanos y el estado de derecho. No obstante, el problema de la inseguridad y la corrupción no es únicamente centroamericano ya que México y Estados Unidos comparten la responsabilidad de resolverlo gracias al tráfico de armas y de drogas. Hasta que no se resuelva plenamente esta externalidad, no podrá integrarse favorablemente el istmo y se verá afectada la movilidad intrarregional de los visitantes.

\section{Conclusiones}

Los recientes avances en la implementación del turismo en América Central han impulsado su proceso de integración regional, y están respaldados por una firme voluntad política y por los progresos registrados en la definición de políticas regionales en otros ámbitos, como medio ambiente y seguridad. Aún hay retos por afrontar para que se consolide plenamente la integración: diseñar un concepto claro, consensuado, a medio o largo plazo, que abata las externalidades generadas por el territorio, completar la unión aduanera centroamericana para que el CA-4 se extienda a todo el istmo, sea una realidad más que un discurso; y poner en práctica las decisiones adoptadas a nivel regional con un máximo de eficacia. La integración regional es un excelente vehículo para promover el desarrollo en Centroamérica y, si se centra en el turismo, se puede hacer crecer económicamente a la región. No obstante, se debe mantener el respaldo de los programas de cooperación internacional.

Es necesaria una mayor inversión en todo el sistema de transporte terrestre para favorecer la movilidad a lo largo del istmo. A pesar de que la Carretera Panamericana es la médula espinal de la movilidad turística, el hecho de que ésta sea una vialidad de sólo un carril y en malas condiciones hace que muchos visitantes decidan no desplazarse por Centroamérica y que no se consolide la integración. Tanto la topografía como las condiciones climáticas centroamericanas repercuten en el estado de la carretera; por lo tanto, urge ampliar el número de carriles y repavimentarle donde sea necesario.

El territorio centroamericano presenta fuertes problemas de fricción del espacio cuando se le recorre por vía carretera. Además, ésta es muy elevada como resultado de la serie de externalidades territoriales y es imperante que se empiecen a gestionar medios de transporte alternativos en el istmo que potencien la integración turística. Actualmente existe Avianca, una aerolínea sudamericana que vincula todas las capitales centroamericanas aunque a partir de costos elevados. Puesto que la extensión territorial del istmo es una ventaja comparativa para la movilidad, se podrían implementar aerolíneas locales de bajo costo o, en su defecto, construir un tren que comunique las capitales. Además, es necesario aumentar las corridas diarias de los autobuses turísticos que vinculan a toda la región.

A pesar de la dificultad para cambiar monedas locales y las ambigüedades fronterizas, la principal externalidad radica en la corrupción y la inseguridad. Con el respaldo del Banco Mundial y la comunidad internacional, cada vez más sectores sociales se unen al diálogo regional para compartir experiencias e identificar soluciones prácticas para abatir ambos problemas. Además, SICA provee apoyo para fortalecer la prevención del delito y la violencia, e incluye inversiones en mejoramiento urbano en Honduras, iniciativas de prevención del delito en El Salvador, y revisiones del gasto público en seguridad ciudadana en Honduras, El Salvador y Guatemala.

$\mathrm{Al}$ estar en proceso de consolidación turística y, año con año, recibir mayor cantidad de visitantes, el futuro del sector demandará que haya mayor movilidad a través del istmo. Así, se deberá incrementar el número de unidades de Ticabus que transitan la Carretera Panamericana y las carreteras se tendrán que ampliar para abatir la fricción del espacio. Además, para que la integración regional en América 
Central esté completa, se debe incorporar a Belize a la ruta de la movilidad; hay una fuerte demanda por parte de los turistas porque la Ciudad de Belize esté comunicada con Guatemala (Tikal) y con Quintana Roo (Chetumal). Ampliar la ruta hacia Belize es un nicho de oportunidad económica para que este país esté incorporado en la dinámica turística y de movilidad.

\section{Bibliografia}

Agencia Española de Cooperación Internacional

2002. Plan de acción para la promoción del turismo sostenible en América Central. Madrid: Agencia

Española de Cooperación Internacional.

Anderson, J., Adey, P., y Bevan, P.

2010. "Positioning place: polylogic approaches to research methodology". Qualitative Research, 10(5): 589-604.

Anselin, L.

2003. "Spatial externalities, spatial multipliers, and spatial econometrics". International Regional Science Review 26(2): 153-166.

Anselin, L., Vargas, A., y Acs, Z.

2000. "Geographic and sectoral characteristics of academic knowledge externalities". Papers in Regional science, 79(4): 435-443.

Bahl, R. W., Martínez, J. y Wallace, S.

1996. The Guatemalan tax reform. Nueva York: Westview Press.

Becattini, G.

2002. Del distrito industrial marshalliano a la «teoría del distrito» contemporánea. Una breve reconstrucción crítica. Investigaciones regionales, (1): 9-32.

Belz, F. y Baumbach, W.

2010. "Netnography as a method of lead user identification". Creativity and Innovation Management, 19(3): 304-313.

Berliant, M., Peng, S. K., y Wang, P.

2002. "Production externalities and urban configuration". Journal of Economic Theory, 104(2): 275-303.

Briedenhann, J. y Wickens, E.

2003. "Tourism routes as a tool for the economic development of rural areas: vibrant hope or impossible dream?" Tourism Management, 25(1): 71-79.

Brohman, J.

1996. "New directions in tourism for third world development". Annals of tourism research, 23(1): 48-70.

Buckley, R.

2003. "The practice and politics of tourism and land management". Nature-based tourism, environment and land management, 1-6.

Cañada, E.

2010. "Tourism in Central America. Social conflict in a new setting". Studies in Contrast; Responsible Tourism. Toronto: Research and Communication for Development.

CARSI

2012. Iniciativa Regional de Seguridad para América Central. [Online]. Versión en línea disponible en: http://www.state.gov/documents/organization/212874.pdf. [Último acceso 03 Oct, 2016]

Das, J. y DiRienzo, C.

2010. "Tourism competitiveness and corruption: A cross-country analysis". Tourism Economics 16(3): 477-492.

Escaith, H.

2001. "Las economías pequeñas de América Latina y el Caribe". Revista de la CEPAL.

Fakeye, P. y Crompton, J.

1991. "Image differences between prospective, first-time, and repeat visitors to the Lower Rio Grande Valley”. Journal of Travel Research 30(1): 10-16.

Ferguson, L.

2010. "Tourism development and the restructuring of social reproduction in Central America". Review of International Political Economy, 17(5): 860-888.

Fernández G. y Ramos, A.

2005. "Patrimonio industrial y rutas turísticas culturales: algunas propuestas para Argentina". Cuadernos de Turismo 15(3): 97-112. 
Fujita, M. y Krugman, P.

2004. "The new economic geography: Past, present and the future". Papers in Regional Science. 83(1): 139-164.

Fujita, M., Krugman, P. y Venables, A.

2001. The spatial economy: Cities, regions, and international trade. Massachussetts: MIT press.

Glaeser, E. y Scheinkman, J.

2000. "Non-Market Interactions". National Bureau of Economic Research. Massachussetts: Cambridge.

Go Central America (2016). Entry Requirements for Central America Travel. [Online]. Versión disponible en: http://gocentralamerica.about.com/od/gettingtherearound/a/Entry-Requirements-For-Central-America-Travel.htm. [Último acceso 05 Nov, 2016].

Griffin, T.

2013. "Research note: A content analysis of articles on visiting friends and relatives tourism, 1990-2010". Journal of Hospitality Marketing \& Management, 22(7): 781-802.

Hagoort, M., Geertman, S., y Ottens, H.

2008. "Spatial externalities, neighbourhood rules and CA land-use modelling". The Annals of Regional Science, 42(1): 39-56.

Hale, C.

2011. “'Resistencia para qué? Territory, autonomy and neoliberal entanglements in the 'empty spaces' of Central America”. Economy and Society, 40(2): 184-210.

Hall, M. y O'Sullivan, V.

1996. "Tourism, political stability and violence (pp. 46-58)". Tourism, crime and international security issues.

Issa, I. y Altinay, L.

2006. "Impacts of political instability on tourism planning and development: the case of Lebanon". Tourism Economics, 12(3): 361-381.

Jiguan, B.

2015. "Promueven la creación del Fodestur". Diario de Centro América. Versión en línea disponible en: http://www.dca.gob.gt/index.php/categoryblog-2/item/38980-promueven-la-creaci\%C3\%B3n-del-fodestur [Último acceso 12 Sep, 2016]

Khadaroo, J. y Seetanah, B.

2008. \#The role of transport infrastructure in international tourism development: A gravity model approach". Tourism management, 29(5): 831-840.

Krugman, $\mathrm{P}$.

2011. The new economic geography, now middle-aged. Regional Studies, 45(1): 1-7.

Laffont, J.

2011. "Externalities" En: Durlauf, N. y L. Blume. The New Palgrave Dictionary of Economics. Segunda Edición. Londres: Palgrave Macmillan.

Lau, T. y Hazari, B.

2011. "Corruption and tourism". Trade and welfare: Theoretical and empirical issues, 159-170.

LeVine, P. y Scollon, R.

2004. Discourse and technology: Multimodal discourse analysis. Georgetown University Press.

Lew, A., y McKercher, B.

2006. "Modeling tourist movements: A local destination analysis". Annals of tourism research, 33(2): 403-423.

Lew, A., Hall, M. y Williams, A.

2008. "Transport and tourism". En Lew,A. (ed.). A Companion to Tourism. Malden: Blackwell Publishing Ltd, 267-306.

Li, T. y McKercher, B.

2016. "Developing a typology of diaspora tourists: Return travel by Chinese immigrants in North America”. Tourism Management, 56: 106-113.

Liu, Z., y Jenkins, C.

1996. "Country size and tourism development: a cross-nation analysis". Sustainable tourism in islands and small states: issues and policies, 90-117.

López, T. y Sánchez S.

2008. "La creación de productos turísticos utilizando rutas enológicas". Pasos. Revista de turismo y patrimonio cultural 6(2): 159-171. 
Lumsdon, L.

2000. "Transport and Tourism: cycle tourism-a model for sustainable development?". Journal of Sustainable Tourism, 104-132.

McKean, J., Johnson, D. y Walsh, R.

1995. "Valuing time in travel cost demand analysis: An empirical investigation". Land Economics, 96-105.

McKercher, B. y Lau, G.

2008. "Movement patterns of tourists within a destination". Tourism Geographies: An International Journal of Tourism Space, Place and Environment 10(3): 355-374.

McKercher, B., Packer, T., Yau, M. y Lam, P.

2003. "Travel agents as facilitators or inhibitors of travel: perceptions of people with disabilities".

Tourism Management, 24(4): 465-474.

Mion, G.

2004. "Spatial externalities and empirical analysis: the case of Italy". Journal of Urban Economics 56(1): 97-118.

Mires, F.

2006. "Los diez peligros de la democracia en América Latina". Cuadernos del CENDES, 23(61): 1-38.

Mora, P.

2013. "Mal estado de carreteras impide integración centroamericana". CB24: Noticias Centroamérica. [Online]. Versión disponible en: http://cb24.tv/mal-estado-de-carreteras-impide-integracion-centroamericana/. [Último acceso 28 Jun, 2016].

Murillo, J. y C. Martínez

2010. "Investigación Etnográfica". Métodos de Investigación Educativa. México: UAM, Xochimilco.

Navalpotro, J.

2001. "Consideraciones sobre las externalidades negativas de la economía de mercado en el contexto de la integración ambiental”. Observatorio Medioambiental 4: 193-203.

NVI Noticias

2011. "Cae Turismo Extranjero por la Corrupción". NVI Noticias [Online]. Versión disponible en: http:// old.nvinoticias.com/principal/47875-cae-turismo-extranjero-corrupcion. [Último acceso 05 Nov, 2016].

Nieto, J.

1996. "Cuando la corrupción invade el tejido social". Nueva sociedad, (145): 148-159.

ONU

2005. Objetivos de Desarrollo del Milenio. Una mirada desde América Latina y el Caribe. Santiago de Chile: Naciones Unidas.

Paasi, A.

2009. "Bounded spaces in a 'borderless world': border studies, power and the anatomy of territory". Journal of Power, 2(2): 213-234.

Pearce, P. y Moscardo, G.

2006. "Domestic and visiting friends and relatives tourism". Tourism business frontiers: Consumers, products and industry, 48-55.

Pigliaru, F., y Lanza, A.

1999. "Why Are Tourism Countries Small and Fast-Growing?". Tourism and sustainable economic development 3(1): 57-69.

Prideaux, B.

2005. "Factors affecting bilateral tourism flows". Annals of Tourism Research, 32(3): 780-801.

Puebla, J.

1998. "Redes, espacio y tiempo". Anales de geografía de la Universidad Complutense 18: 65-86.

Reid, R.

2007. Central America on a Shoestring. Footscray: Lonely Planet Publications Ltd.

Robinson, W.

2003. Transnational conflicts: Central America, social change and globalization. Philadelphia: Verso.

Rodríguez, M. y Dombrow, J.

2016. “PPor qué la dolarización frenó el crecimiento en El Salvador?” El Faro Académico. Versión en línea disponible en: https://elfaro.net/es/201601/academico/17865/. [Último acceso: 28 Ene, 2018].

Romanelli, E. y Khessina, O.

2005. "Regional industrial identity: Cluster configurations and economic development". Organization Science, 16(4), 344-358.

Rudolph, C.

2005. "Sovereignty and territorial borders in a global age". International studies review, 7(1): 1-20. 
Segovia, A.

2005. Integración real y grupos de poder económico en América Central: implicaciones para el desarrollo y la democracia de la región. San Jose: Friedrich Ebert Stiftung.

SICA

2013. Central American Integration System (SICA.) SICA, San José, Costa Rica. Versión en línea disponible en: https://www.mea.gov.in/Portal/ForeignRelation/Central_American_Integration_System_SICA_. pdf. [Último acceso: 28 Jun, 2016].

Solís, W.

2014. "Integración del Mercado Común Centroamericano (MCCA)". Revista Electrónica de Investigación en Ciencias Económicas: "Abriendo Camino al Conocimiento". 28-55.

Sunkel, O.

1998. "Desarrollo e integración regional: ¿otra oportunidad para una promesa incumplida?". Revista de la CEPAL, (Extraordinario).

Suzuki, F.

2010. "A distance friction minimization approach in data envelopment analysis: a comparative study on airport efficiency". European Journal of Operational Research 207(2): 1104-1115.

Tedlock, B.

2010. "From participant observation to the observation of participation: The emergence of narrative ethnography". Journal of anthropological research, 47(1): 69-94.

Timothy, D.

1995. "Political boundaries and tourism: Borders as tourist attractions". Tourism Management 16(7): 525-532.

Tosun, C. y D. Timothy

2001. "Shortcomings in planning approaches to tourism development in developing countries: the case of Turkey". International Journal of Contemporary Hospitality Management, 13(7): 352-359.

Trip Advisor

2016. "Safety". Central America Forum. [Online]. Versión disponible en: https://www.tripadvisor.co.nz/ ShowTopic-g294475-i3217-k9953052-Safety-El_Salvador.html\#78852560. [Último acceso 05 Nov, 2016]

Truong, T. y Henscher, D.

1985. "Measurement of travel time values and opportunity cost from a discrete-choice model". The Economic Journal 65(378): 438-451.

Ullate, J.

2014. The Central American Integration System (SICA) and Its Efforts for the Harmonization of Medicine Regulation. Versión en línea disponible en: http://lifesciences.thomsonreuters.com/sites/ls/files/m/ pdf/cri-sica_whitepaper.pdf. [Último acceso: 28 Jun, 2016].

Wang, $\mathrm{N}$.

1999. "Rethinking authenticity in tourism experience". Annals of tourism research, 26(2): 349-370.

Wikitravel

2016. Central America [blog]. Versión disponible en: http://wikitravel.org/en/Central_America. [Último acceso 05 Nov, 2016].

Zakrisson, I. y Zillinger, M.

2012. "Emotions in motion: tourist experiences in time and space". Current Issues in Tourism 15(6): 505-523.

\section{Notas}

1 Este tipo de turistas prefieren lugares menos turísticos, disfrutan de los destinos innovadores o poco concurridos (si es que ellos existen aún en la dinámica global), conocer a gente local y extranjera durante el viaje y son flexibles en cuanto a sus patrones de movilidad.

2 Polilogismo es la creencia de que diferentes grupos de personas razonan de formas fundamentalmente diferentes. En la investigación turística, se atribuye que hay diferentes formas de "lógica" en los diversos grupos de visitantes, que actúan y viven el espacio a partir de preferencias basadas en género, clase, cercanía cultural o período de tiempo.

3 Aquellos individuos o entes que consumen más que una parte equitativa de un recurso, o no afrontan una parte justa del costo de su producción.

Recibido:

$28 / 01 / 2017$

Reenviado:

$03 / 02 / 2018$

Aceptado:

$12 / 02 / 2018$

Sometido a evaluación por pares anónimos 